\title{
DESEMPENHO DO MODELO DE SUPERFÍCIE NOAH PARA SIMULAÇÕES DE SOLO EM UMA REGIÃO COM AGRICULTURA
}

\author{
Juliana B. Gonçalves ${ }^{1}$, Débora R. Roberti ${ }^{1}$, Andréa U. Timm² ${ }^{2}$ Gervásio A. Degrazia ${ }^{1}$ \\ ${ }^{1}$ Departamento de Física/Lab. Micrometeorologia/UFSM, Santa Maria, RS, Brasil \\ ${ }^{2}$ Faculdade da Serra Gaúcha, FSG, Caxias do Sul, RS, Brasil
}

1ju-1302@hotmail.com

\section{RESUMO}

O objetivo deste estudo é avaliar o desempenho do modelo de superfície Noah através da simulação de dados de solo e compará-los com dados observados em uma região com agricultura no sul do Brasil. A partir dos resultados, observou-se que o modelo Noah representa satisfatoriamente as quantidades avaliadas. Porém, necessita de algumas parametrizações, uma vez que o Noah simula a temperatura do solo como se a radiação incidisse diretamente na superfície.

\begin{abstract}
The aim of this study is to evaluate the performance of the model surface Noah by simulating soil data and compare them with observed data in a region with agriculture in southern Brazil. The results showed that the model is satisfactory according to the Noah. However, it requires some parameterization, since Noah simulates the soil temperature as focussing radiation directly to the surface without regarding to whether there is coverage on the ground.
\end{abstract}

\section{INTRODUÇÃO}

A escolha deste tema foi consequência do entendimento de que a precisão na estimativa das variáveis de solo está diretamente associada à precisão da previsão meteorológica. Neste 
contexto, entende-se que previsões representativas auxiliam no desenvolvimento do uso da água, tão relevantes para áreas agrícolas. Este estudo foi desenvolvido utilizando o modelo de superfície Noah, responsável por descrever a hidrodinâmica e termodinâmica do sistema solo-atmosfera.

\section{DADOS E METODOLOGIA}

Para as simulações com o modelo Noah, foram utilizados dados coletados por uma estação meteorológica do INMET localizada no município de Cruz Alta/RS (28³6’S, 5340’O) na Fundação Centro de Experimentação e Pesquisas Fecotrigo (FUNDACEP). O período abrangido pelo estudo foi de 01 de fevereiro de 2009 até 31 de janeiro de 2010. Foram utilizadas médias diárias de 30 minutos das variáveis meteorológicas para entrada no modelo. O modelo de superfície Noah é responsável pela descrição da hidrodinâmica e termodinâmica do sistema solo-atmosfera e pela estimativa das variáveis que caracterizam os processos físicos. Este modelo começou a ser desenvolvido na década de 1990 para ser utilizado na previsão numérica do tempo na Oregan State University (OSU). O Noah evoluiu recebendo novas parametrizações físicas (Chen et al., 1996). A temperatura do solo é estimada através do esquema de Crank-Nichlson na forma da camada integrada a partir da Equação (1),

$$
\mathrm{C}(\theta) \frac{\partial \mathrm{T}}{\partial \mathrm{t}}=\frac{\partial}{\partial \mathrm{z}}\left(\mathrm{K}_{\mathrm{t}}(\theta) \frac{\partial \mathrm{T}}{\partial \mathrm{z}}\right)
$$

sendo a capacidade térmica volumétrica $\mathrm{C}\left(\frac{\mathrm{J}}{\mathrm{m}^{3} \mathrm{~K}}\right)$ e a condutividade térmica $\mathrm{K}_{\mathrm{t}}\left(\frac{\mathrm{W}}{\mathrm{mK}}\right)$ formuladas como função do conteúdo de água no solo volumétrico $(\theta)$. No Noah, o transporte de água no solo segue a forma da difusividade da Equação de Richards,

$$
\frac{\partial \theta}{\partial \mathrm{t}}=\frac{\partial}{\partial \mathrm{z}}\left(\mathrm{D}(\theta) \frac{\partial \theta}{\partial \mathrm{z}}\right)+\frac{\partial \mathrm{k}}{\partial \mathrm{z}}+\mathrm{F}_{\theta}
$$

Sendo $\theta\left(\frac{\mathrm{m}^{3}}{\mathrm{~m}^{3}}\right)$ o conteúdo volumétrico de água no solo, $\mathrm{D}\left(\frac{\mathrm{m}}{\mathrm{s}^{2}}\right)$ a difusividade da água no solo, $\mathrm{k}\left(\frac{\mathrm{m}}{\mathrm{s}}\right)$ a condutividade hidráulica e $\mathrm{F}_{\theta}$ são as fontes e sumidouros de água no solo. Integrando-se a Eq.(2) e expandindo-se $\mathrm{F}_{\theta}$, obtém-se o conteúdo volumétrico de água para cada uma das quatro camadas de solo simuladas. 


\section{RESULTADOS}

A Figura1 mostra a comparação entre as temperaturas do solo observadas e simuladas para o período de 01/fev/09 até 31/jan/10 obtidas experimentalmente e simuladas pelo Noah. Podese observar que o modelo representa satisfatoriamente esta variável. Algumas diferenças são notadas entre os meses de maio a agosto (diferença máxima de $6^{\circ} \mathrm{C}$ ). A temperatura do solo não alcança valores elevados quando há muita precipitação.

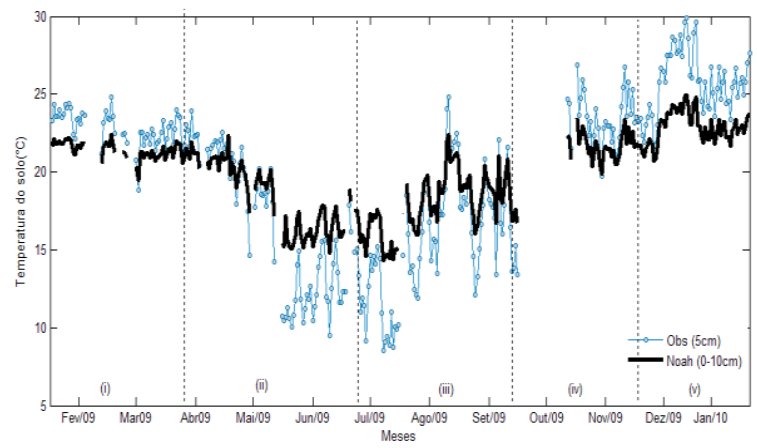

Figura 1 - Média diária da temperatura do solo observada a $5 \mathrm{~cm}$ e simulada na camada entre $0-10 \mathrm{~cm}$ de profundidade. Os pontilhados verticais indicam os períodos com diferentes manejos do solo (i) soja, (ii) pousio, (iii) aveia preta, (iv) pousio e (v) soja.

Os valores para a umidade do solo observada e simulada para diferentes profundidades estão indicadas na Figura 2. O conteúdo de água no solo foi maior no final do mês de julho, em setembro e entre os meses de novembro e dezembro, onde a precipitação foi maior. Os resultados do modelo mesmo nas camadas mais profundas assumem valores de acordo com a precipitação (Fig.3).

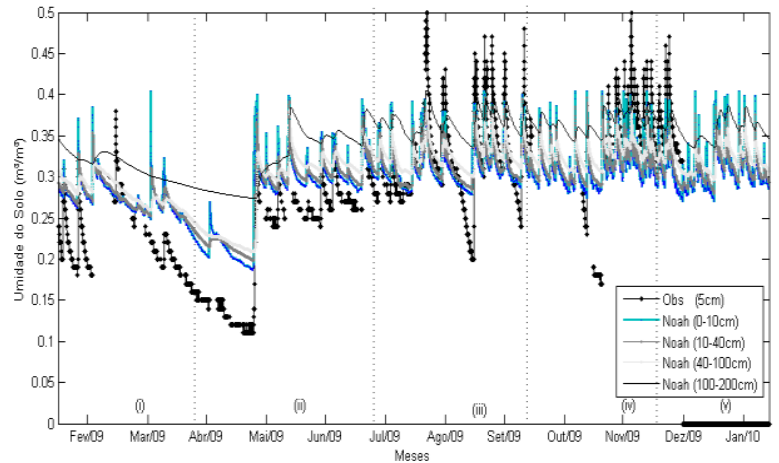

Figura 2 - Média diária da umidade do solo observada a $5 \mathrm{~cm}$ e simulada nas camadas $(0-10 \mathrm{~cm}$; $10-40 \mathrm{~cm} ; 40-100 \mathrm{~cm}$ e $100-200 \mathrm{~cm}$ de profundidade). Os pontilhados verticais indicam os períodos com diferentes manejos do solo (i) soja, (ii) pousio, (iii) aveia preta, (iv) pousio e (v) soja. 
A Figura 3 mostra a precipitação acumulada para o período estudado. Esta quantidade apresenta maiores acumulados a partir do mês de maio até o mês de janeiro/10. E valores menores entre fevereiro e abril.

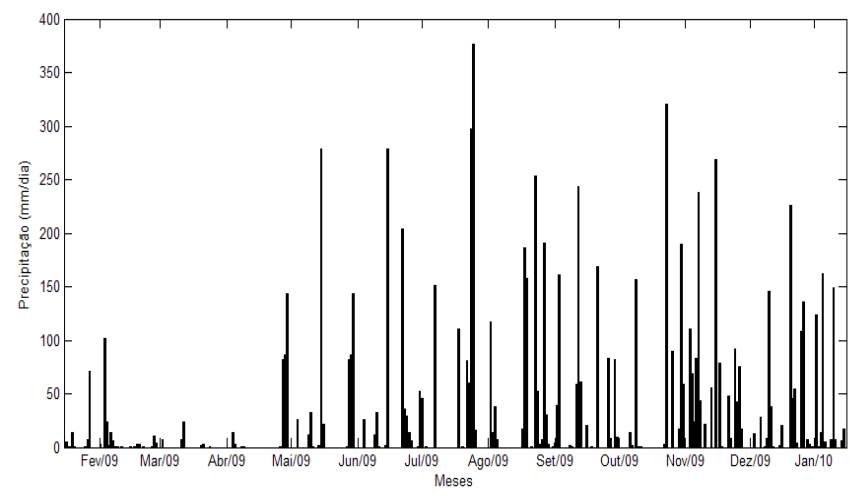

Figura 3 - Precipitação diária acumulada para o período de estudo.

\section{CONCLUSÕES}

Os resultados das análises observadas de solo foram utilizados em confronto com as simulações e observou-se um bom desempenho do modelo Noah tanto para a temperatura quanto para a umidade do solo. Como o Noah simula a temperatura do solo considerando que a radiação incidente atinge diretamente a superfície, surge a necessidade de novas parametrizações no modelo para o cálculo dessa variável.

\section{REFERÊNCIAS}

CHEN, F. et al. Modeling of land-surface evaporation by four schemes and comparison with five observations. Journal of Geophysical Research, v.101, n.D3, p.7251-7268, 1996. 